\title{
Impact of telehealth on general practice contacts: findings from the whole systems demonstrator cluster randomised trial
}

\author{
Martin Bardsley ${ }^{1 *}$, Adam Steventon $^{1}$ and Helen Doll ${ }^{2,3}$
}

\begin{abstract}
Background: Telehealth is increasingly used in the care of people with long term conditions. Whilst many studies look at the impacts of the technology on hospital use, few look at how it changes contacts with primary care professionals. The aim of this paper was to assess the impacts of home-based telehealth interventions on general practice contacts.

Method: Secondary analysis of data from a Department of Health funded cluster-randomised trial with 179 general practices in three areas of England randomly assigned to offer telehealth or usual care to eligible patients. Telehealth included remote exchange of vitals signs and symptoms data between patients and healthcare professionals as part of the continuing management of patients. Usual care reflected the range of services otherwise available in the sites, excluding telehealth. Anonymised data from GP systems were used to construct person level histories for control and intervention patients. We tested for differences in numbers of general practitioner and practice nurse contacts over twelve months and in the number of clinical readings recorded on general practice systems over twelve months.
\end{abstract}

Results: 3,230 people with diabetes, chronic obstructive pulmonary disease or heart failure were recruited in 2008 and 2009. 1219 intervention and 1098 control cases were available for analysis. No statistically significant differences were detected in the numbers of general practitioner or practice nurse contacts between intervention and control groups during the trial, or in the numbers of clinical readings recorded on the general practice systems.

Conclusions: Telehealth did not appear associated with different levels of contact with general practitioners and practice nurses. We note that the way that telehealth impacts on primary care roles may be influenced by a number of other features in the health system. The challenge is to ensure that these systems lead to better integration of care than fragmentation.

Trial registration number: International Standard Randomised Controlled Trial Number Register ISRCTN43002091.

Keywords: Telemedicine, Telemonitoring, General practice, Workload, Chronic disease

\section{Background}

The use of telehealth to allow patients with long-term health conditions to monitor vital signs and transfer readings to health professionals working remotely is increasingly being advocated as a way of delivering higher quality care more efficiently for better management of people with long term conditions [1]. Very often, one of the benefits of telehealth is perceived to be its potential

\footnotetext{
* Correspondence: martin.bardsley@nuffieldtrust.org.uk

${ }^{1}$ The Nuffield Trust, 59 New Cavendish Street, W1G 7LP London, UK

Full list of author information is available at the end of the article
}

impact on the use of hospital care. Relatively little attention has been placed on its impacts on primary care services such as general practices. Yet these are important determinants of patient experience and quality of care and the costs of providing primary and community health care to populations with long-term health conditions can be almost as large as hospital costs [2]. The impact of these technologies on primary care is therefore an important element in understanding the opportunities and potential barriers to introducing telehealth [3].

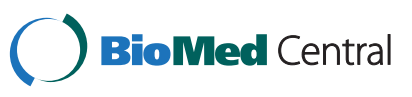


Two alternative hypotheses exist for the potential impact of telehealth on primary care. First, telehealth might reduce the need for support from primary care; for example, because problems are detected earlier, patients develop better self-care skills, or there is less need to undertake measurements such as weight in general practice. Telehealth may also serve to buffer the general practice in situations where the professionals who are monitoring patients as part of the telehealth service are distinct from general practice and have the authority to provide clinical care, for example by adjusting treatment and/or reassuring patients. In some instances, the patient contact may go directly to the hospital consultant [4]. Conversely, telehealth might increase the need for support from primary care, if the extra clinical information obtained through telehealth prompts calls for intervention from professionals. This could be due to abnormal readings that would in the absence of monitoring have returned to a normal range; or due to heightened awareness from patients.

Few studies have addressed the impact of telehealth on primary care. Some small studies have observed indications of time savings for general practices $[5,6]$ and one noted a non significant reduction in home nursing visits and a reduction in hospital admission [7]. A randomised trial of 40 patients' with moderate to severe Chronic Obstructive Pulmonary Disease (COPD) found that telemonitoring did not change the rate of emergency hospital visits but did reduce primary care contacts for chest problems [8]. A more recent randomised trial of remote blood pressure monitoring in 401 patients with hypertension found improved control of blood pressure but increased general practitioner and nurse consultations $[9,10]$. The Whole Systems Demonstrator programme aimed to address weaknesses in the evidence base for the effectiveness of telehealth in people with COPD, heart failure and diabetes, through a wide-ranging evaluation in three sites in England, UK (Cornwall, Kent and Newham) [11]. Over 3,000 patients were recruited and received telehealth or usual care on the basis of randomised allocations made at the general practice level. The first published results showed fewer urgent and unplanned ('emergency') hospital admissions among telehealth patients than usual-care controls [12], though this appeared to have been linked with increases in emergency admissions among the control group following the start of the trial. Overall savings through reduced hospital activity over one year were not statistically significant. Though reduced mortality rates were seen, there was no overall improvements in quality of life among survivors [13], and the intervention was not found to be cost effective at realistic willingness-to-pay thresholds [2].

In this paper, we describe secondary analysis of the General Practice (GP) data sets amassed for the Whole
Systems Demonstrator evaluation. The focus here is on the impact of telehealth on primary care physicians (general practitioners) and on nurses based in general practices (practice nurses). To assess this we compared changes between intervention and control groups in terms of the frequency of visits to general practitioners and practice nurses. We also addressed the number of times clinical readings relevant to the management of patients were recorded on the general practice systems.

\section{Methods}

The protocol for the Whole Systems Demonstrator evaluation has previously been described and included plans to undertake secondary analysis of primary care use $[11,12]$. The study randomised general practices to offer either telehealth or usual care to eligible patients (criteria in Table 1) with COPD, diabetes or heart failure. Control patients received usual care and were offered telehealth at the end of the twelve-month trial period if they were still eligible at that point.

\section{Telehealth intervention}

The pragmatic trial design meant that there was flexibility for local teams to develop their own telehealth services. Therefore, choices of telehealth devices and monitoring systems varied between the three trial sites and there was no attempt to standardise across sites. Sites used different protocols for allocating peripheral devices but across all sites the critical devices per

\section{Table 1 Eligibility for the trial}

\begin{tabular}{ll}
\hline Practice & All practices within the geographical areas covered by \\
characteristics & the sites (Cornwall, Newham and Kent) were eligible \\
& and were invited to participate in the trial by letter. \\
& Each practice that accepted the invitation to \\
& participate was allocated to an intervention or control \\
& group via a centrally-administered minimisation \\
& algorithm that aimed to ensure that the groups of \\
& practices were similar in terms of practice size, \\
& deprivation index, proportion of non-white patients, \\
& prevalence of diabetes, COPD and heart failure, and \\
& site (Cornwall, Kent and Newham). \\
\hline Patient & Within each practice, patients aged 18 or over were \\
characteristics & primary or secondary care for COPD, heart failure or \\
& diabetes. \\
& Eligibility was not conferred on the basis of formal \\
clinical assessment of disease severity. Instead \\
patients were deemed eligible on the basis of either \\
(i) their inclusion on the relevant Quality Outcomes \\
Framework register in primary care, (ii) a confirmed \\
medical diagnosis in primary or secondary care \\
medical records as indicated by general practice Read \\
Codes or ICD-10 codes, or (iii) confirmation of disease \\
status by a local clinician (i.e. general practitioner or \\
community matron) or by their hospital consultant. \\
Patients were not excluded on the basis of additional \\
physical co-morbidities. However, the patient's home \\
had to be suitable for the installation of telehealth. \\
\hline
\end{tabular}


condition were pulse oximeters (for COPD), blood glucose monitors (for diabetes) and weighing scales (for heart failure). In addition, almost all intervention participants received blood pressure monitors. Participants were asked to take clinical readings up to five days per week at the same time each day. In addition symptom questions and educational messages were transmitted to participants, either via the telehealth base unit or using a set-top box connected to a television. At the end of each session, data were transmitted to monitoring centres via secure servers. These centres were staffed by local health organisations. Sites used a variety of approaches to respond to triggers generated by worsening patterns in telehealth readings. These ranged from an approach based on locating specialist nurses in monitoring centres to routing alerts to community-based staff, such as community matrons. In one of the two Kent Primary Care Trusts, warning signs from telehealth were routed direct to general practices for their response.

\section{Data sets}

Information about service activity was derived from extracts from operational information systems and in particular exploiting the system of coded data on computerised general practice systems. Though there are national standard coding systems available and widely used, there can be differences between practices in the specify codes used. All general practices participating in the trial were asked to share data for the whole of their adult practice population, including registration and encounter dates, diagnoses, test results and prescriptions. Data were "pseudonymised" before being transferred to the research team, so that patientidentifiable fields could be removed and a unique patient identifier (the "NHS number") encrypted. Pseudonymised NHS numbers were used to link the general practice data to hospital data sets which included information on inpatient stays, outpatient attendances and emergency visits. The research team created person-level data sets to summarise all key health care contacts experienced by the participants over several years. The approach of using pseudonyms in secure environments is regarded by the National Information Governance Board as appropriate technique for these types of study where patient consent across a whole population is not feasible. The study was approved by Liverpool Research Ethics Committee (ref: 08/H1005/4).

\section{Study cohort and end points}

This study was restricted to participants (linked to GP data) that were enrolled into the trial before the planned recruitment termination date (30 September 2009). The trial enrolment date was taken as the date of telehealth installation for intervention patients, and as the date of the initial project team visit for controls. Further, we required that patients had a continuous prior record of registration with general practices within the data sets, spanning the two years prior to the trial and the period of the trial itself (excluding patients that died).

Analysis was based on comparing activity over 12 months from the enrolment dates, at the person level. General practice workload was based on the number of contacts with a principal general practitioner, locum or registrar (coded as staff types $\mathrm{A}, \mathrm{B}$ or $\mathrm{C}$ ); and practice nurses in primary care (staff type D06). In both cases, contacts were obtained from the computerised practice systems and included all locations (GP Surgery, Home Visit, Telephone etc.). We also studied the number of times test results were recorded within the general practice records specifically for glycosylated haemoglobin A1C, weight, blood oxygen levels and respiratory flow (relevant Read codes in Additional file 1). These metrics were chosen as they were akin to the readings that were regularly made by patients receiving telemonitoring. A variable was created based on the count of different readings obtained per person.

\section{Statistical analysis}

We examined the similarity of intervention and control patients at baseline using the standardised difference, defined as the difference in sample means as a proportion of the pooled standard deviation [14]. Previous studies have used a standardised difference of $10 \%$ as a threshold to denote meaningful difference in baseline variables [15].

To provide information on the generalisability of trial results, we tested for differences between the general practice contact rates of trial participants and those of the wider adult population (among the practices that provided data for the evaluation). In this analysis, these comparisons were based on the number of contacts in 2009/10 and were adjusted for age band and sex using Poisson regression. As not all patients were alive and registered with a practice for the whole of the 2009/ 10 year, we annualised rates by including an offset in the Poisson regression.

Individuals were analysed on an "intention-to-treat" basis, i.e. based on the randomised treatment allocations of the general practices, and regardless of subsequent withdrawal from the trial. We used two methods to compare intervention and control groups. The first used a difference-in-difference method to assess whether the number of contacts and recorded clinical readings increased more quickly or more slowly among the intervention group than controls, from the year before enrolment to the year after. This was done using ordinary least squares regression, with the output relating to an absolute number of admissions. The second analysis made a direct comparison of numbers of contacts and 
clinical readings experienced within the twelve-month trial period. This was done using Poisson regression, with exponentiation of the model coefficients producing an incidence rate ratio. As in the primary analysis [12], three versions of the incidence rate ratio were produced, with different forms of case-mix adjustment (Table 2).

We expected that contact rates would be more highly correlated for patients registered at the same practice than at different practices. Therefore, all of our models reflected intracluster correlation at the practice level, using multilevel models with random effects at the practice level [17].

\section{Results}

\section{Patient recruitment}

249 out of 254 practices agreed to share routine data from their systems for the evaluation, although data extraction was not possible for a further 5 practices for technical reasons linked with the clinical operating systems used. The data sets obtained spanned more than four years (April 2006 to September 2010) and contained almost one billion records. Ultimately, 1,625 control and 1,605 intervention

\section{Table 2 Three forms of case-mix adjustment used in} analysis

\begin{tabular}{ll}
\hline Unadjusted & The simplest models, although accounting for the effect \\
& of clustering, used no additional case-mix adjustment. \\
\hline Adjusted & These models additionally controlled for residual \\
imbalances in a set of baseline characteristics. This set \\
included age, sex, ethnicity, site, number of chronic health \\
conditions, principal long-term condition (diabetes, \\
chronic obstructive pulmonary disease or heart failure), an \\
area-based socioeconomic deprivation score (national \\
quartiles of the Index of Multiple Deprivation 2007), and a \\
metric corresponding to the endpoint (e.g., general \\
practitioners contacts) calculated over several periods \\
within the two years prior to recruitment. \\
The number of chronic health conditions was a count of \\
diagnoses recorded on inpatient data over the three \\
years prior to starting the trial. Principal long-term \\
conditions were assigned using a pragmatic approach \\
according to published criteria [16]. \\
More complex case-mix adjustment was conducted using \\
the Combined Predictive Model [16] a standard \\
instrument designed to estimate the probability that an \\
individual will experience an emergency hospital \\
admission in a future twelve month period. The Combined \\
Model score uses 72 variables covering age, sex, recorded \\
health conditions, prior hospital use and prescribing, but \\
not primary care contacts. These variables are sourced \\
from general practice and hospital administrative data. \\
Where a general practice did not grant approval to extract \\
data for the evaluation, or where scores could not be \\
calculated, scores were imputed for its patients based on \\
the available information, which included age, sex and the \\
hospital variables. Single imputation was used based on \\
linear regression on the logit scale. When used in the case- \\
mombined start date. \\
model
\end{tabular}

patients were recruited into the telehealth part of the trial from 179 general practices (for full Consort flow diagram see Steventon et al. [12]).

The analysis of general practice use was restricted to 1,219 intervention and 1,098 control participants recruited before September 2009 and with a continuous general practice registration (76\% of intervention patients and $68 \%$ of controls). Excluded participants were not statistically different from those included in terms of age at baseline, sex and number of chronic conditions. However, included participants were more likely to come from Newham $(31.0 \%$ of included patients compared with $26.7 \%$ of the overall sample), and from the $20 \%$ most socioeconomically deprived areas (21.4\% of patients compared with $19.5 \%)$. Of patients included, a full Combined Predictive Model score could be computed for 2,238 patients (96.6\%); scores were imputed for the remainder using the method described in Table 2.

The routine data sets showed that, among the general population in 2009/10, the consultation rates for trial participants were higher than the general population (incidence rate ratio 2.00, $\mathrm{p}<0.001$ ) and showed no clear trend by age. Similar patterns were found for practice nurse contacts (incidence rate ratio 1.97, p < 0.001).

\section{Baseline differences}

Intervention and control patients were similar at baseline (Table 3), with only a few standardised differences greater than $10 \%$. The largest standardised differences related to the proportion of people living in the most socioeconomically deprived fifth of the population $(8.7 \%$ intervention and $5.1 \%$ of controls, standardised difference $14.3 \%)$ and to long-term health conditions $(27.1 \%$ of intervention patients had diabetes as their principal condition compared with $22.6 \%$ of controls, standardised difference 10.4\%).

Before the start of the trial, rates of general practitioner contact were similar for the intervention and control groups, at around one visit every 6 weeks ( 8.8 vs. 9.0 visits per person per year, standardised difference $2.0 \%$ ). Some patients (constituting $8.1 \%$ of control patients) had over 20 visits with a general practitioner during the year before the start of the trial, while $4.5 \%$ had no recorded contacts on general practice information systems. The average number of practice nurse contacts during the year before enrolment was slightly lower in the intervention than control group (5.3 contacts per person per year versus 6.1, standardised difference $10.2 \%)$. Over both groups $15.2 \%$ of patents had no recorded practice nurse contacts whilst $5.3 \%$ of participants had over 20 contacts in the year before the start of the trial.

Figure 1 shows trends in general practitioner and practice nurse contacts by month, without adjusting for 
Table 3 Baseline characteristics of intervention and controls groups (data are \% of group unless otherwise specified)

\begin{tabular}{|c|c|c|c|}
\hline & Control & Intervention & $\begin{array}{r}\text { Standardised } \\
\text { difference (\%) }\end{array}$ \\
\hline Number in group & 1098 & 1219 & \\
\hline Number of practices & 80 & 82 & \\
\hline $\begin{array}{l}\text { Number of patients } \\
\text { per practice } \\
\text { (median (range)) }\end{array}$ & 10 (1 to 62$)$ & $8(1$ to 76$)$ & \\
\hline \multicolumn{4}{|c|}{ Index long-term condition } \\
\hline $\begin{array}{l}\text { Chronic Obstructive } \\
\text { Pulmonary Disease }\end{array}$ & 47.4 & 45.0 & -4.8 \\
\hline Diabetes & 22.6 & 27.1 & 10.4 \\
\hline Heart failure & 30.0 & 27.9 & -4.6 \\
\hline $\begin{array}{l}\text { Number of chronic } \\
\text { health conditions } \\
\text { (mean (SD)) }\end{array}$ & $1.9(1.8)$ & $1.8(1.8)$ & -3.9 \\
\hline \multicolumn{4}{|l|}{ Site } \\
\hline Cornwall & 32.6 & 36.5 & 8.2 \\
\hline Kent & 36.2 & 32.7 & -7.2 \\
\hline Newham & 31.2 & 30.8 & -1.0 \\
\hline Age (mean (SD)) & $70.8(11.8)$ & $69.7(11.6)$ & -9.3 \\
\hline Aged under 65 & 28.7 & 30.1 & 3.1 \\
\hline Aged 65-74 & 31.4 & 34.9 & 7.5 \\
\hline Aged 75-84 & 30.9 & 27.4 & -7.7 \\
\hline Aged $85+$ & 9.0 & 7.5 & -5.3 \\
\hline Female (\%) & 40.4 & 41.1 & 1.3 \\
\hline
\end{tabular}

Ethnicity

\begin{tabular}{lrrr} 
White & 71.3 & 71.8 & 1.0 \\
Non-white & 13.2 & 12.3 & -2.7 \\
Unknown & 15.5 & 15.9 & 1.2 \\
\hline Area-level deprivation & $29.8(13.8)$ & $28.8(14.9)$ & -6.9
\end{tabular}
$(\text { mean }(\mathrm{SD}))^{*}$

\begin{tabular}{lrrr}
$1^{\text {st }}$ quartile & 5.1 & 8.7 & 14.3 \\
$2^{\text {nd }}$ quartile & 15.4 & 15.5 & 0.2 \\
$3^{\text {rd }}$ quartile & 31.8 & 32.6 & 1.9 \\
$4^{\text {th }}$ quartile & 47.7 & 43.2 & -9.2 \\
\hline GP visits per person & $9.0(7.6)$ & $8.8(6.8)$ & -2.0 \\
(prior year) (mean (SD)) & & & \\
None & 4.5 & 3.5 & -4.8 \\
$1-5$ & 35.2 & 33.1 & -4.6 \\
6-10 & 29.0 & 30.5 & 3.4 \\
$11-20$ & 23.2 & 26.4 & 7.4 \\
$>$ 20 & 8.1 & 6.5 & -6.3 \\
\hline Practice nurse contacts & $6.1(8.1)$ & $5.3(7.8)$ & -10.2 \\
per person (prior year) & & & \\
(mean (SD)) & & & \\
None & 14.8 & 15.5 & 2.1 \\
1-5 & 51.1 & 57.1 & 12.1 \\
6-10 & 16.4 & 14.8 & -4.5
\end{tabular}

Table 3 Baseline characteristics of intervention and controls groups (data are \% of group unless otherwise specified) (Continued)

\begin{tabular}{lrrr}
\hline $11-20$ & 11.9 & 7.8 & -13.9 \\
$>20$ & 5.8 & 4.8 & -4.4 \\
\hline Combined Model & $27.0(20.2)$ & $26.1(20.1)$ & -4.3 \\
score (mean (SD))** & & & \\
Low risk & 14.6 & 15.9 & 3.5 \\
Moderate risk & 30.0 & 32.1 & 4.5 \\
High risk & 44.3 & 41.6 & -5.6 \\
Very high risk & 11.1 & 10.5 & -1.9 \\
\hline
\end{tabular}

$\mathrm{SD}=$ Standard deviation.

${ }^{*} \mathrm{n}($ controls $)=1096, \mathrm{n}$ (intervention $\left.=1214\right)$. First quartile is least deprived, fourth quartile is most deprived.

${ }^{* *} \mathrm{n}$ (controls) $=1,047 ; \mathrm{n}$ (intervention) $=1,191$. Risk categories denote top proportions of site population: very high risk (0.5\%), high risk (0.5-5\%), moderate risk (5-20\%), and low risk (20-100\%).

baseline covariates. The numbers of visits per person were stable before the start of the trial. During the 12 months of the trial, the number of visits fell slightly for the whole cohort. However, a number of patents died during the 12 months (4.9\% of intervention patients included in this sample, compared with $9.1 \%$ of controls in the sample). The average quarterly rate of general practitioner contacts for survivors was 2.14 for intervention patients at the end of the trial and 2.26 for controls. Mortality may therefore explain some of the slight fall in visits.

\section{Analyses of differences in contact rates during the trial period}

As previously stated, the difference-in-difference analysis and Poisson regressions took clustering into account; the intracluster correlation coefficient (ICC) for general practitioner contacts was estimated to be 0.184 .

During the 12 month trial period, the number of general practitioner contacts in the intervention group (mean 8.99, standard deviation 7.00) was similar to that in the control group (mean 8.85, standard deviation 8.16), see Table 4 . When compared with the contacts during the 12 months before the trial started, the difference-in-difference estimate indicated that contacts rose more quickly among the intervention than control group, by 0.29 contacts per head. However, this was not statistically significant $(\mathrm{p}=0.465)$ with an adjusted incidence rate ratio was 1.04 (95\% confidence interval, 0.95 to 1.30 , Table 5). Differences did not reach statistical significance after case-mix adjustment using Poisson regression. While the estimates for rise in contacts were higher for practice nurses than for general practitioners (difference-in-difference estimate 0.45 contacts per head; 


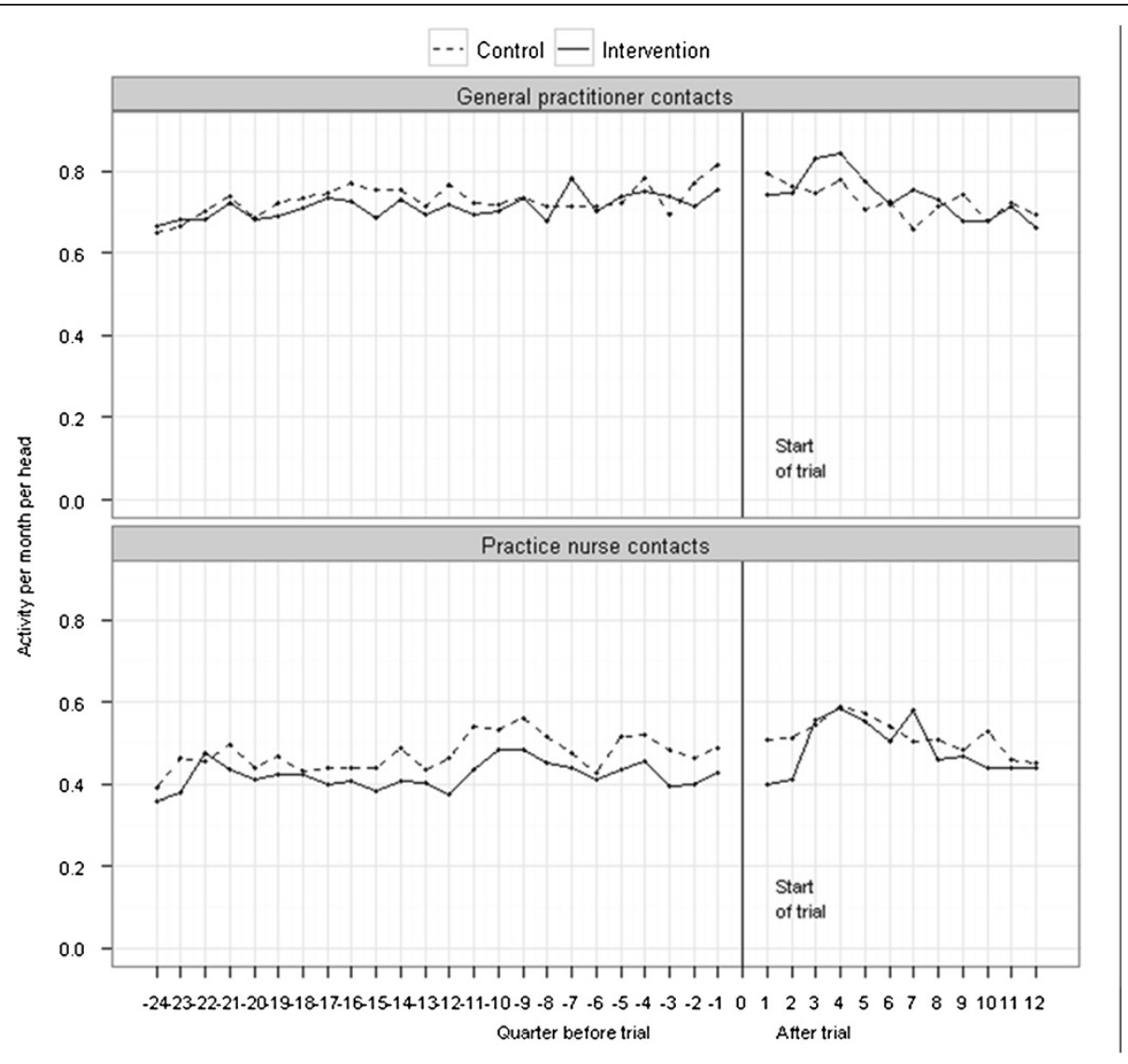

Figure 1 Crude monthly rates of contact.

adjusted incidence rate ratio $1.04,95 \%$ CI 0.82 to 1.30 ), they were not statistically significant.

The number of clinical readings summed across the four clinical domains was similar between intervention and control groups in the 12 months before the trial (2.73 versus 2.67 readings per person). The differencein-difference estimate indicated that the number of readings increased more quickly among the intervention than control group (by 0.11 readings per head) but this was not statistically significant $(p=0.414)$. The adjusted Poisson analysis reported very similar rates of clinical readings during the twelve months of the trial (adjusted incidence rate ratio $1.00,95 \% \mathrm{CI}, 0.90$ to 1.12 , $\mathrm{p}=0.931)$.

\section{Discussion}

\section{Summary}

This study addressed a weakness in the evidence base about the impact of telehealth on primary care by exploiting operational data sets from 179 general practices. On average, over the twelve months before the start of the Whole Systems Demonstrator trial, participants had 8-9 consultations with general practitioners, which was substantially higher than for the general population of the three sites during 2009/10 and more consultations than reported in other studies for people of a similar age [18].

We found no evidence to support the theory that telehealth alters rates of contact with general practices.

Table 4 Unadjusted rates of contacts and readings (figures are numbers per patient over twelve months (SD))

\begin{tabular}{|c|c|c|c|c|c|c|}
\hline & \multicolumn{2}{|c|}{ Before } & \multicolumn{2}{|c|}{ After } & \multicolumn{2}{|c|}{ Difference-in-difference } \\
\hline & Control & Intervention & Control & Intervention & Estimate & $\mathbf{P}$ \\
\hline GP contacts & $8.98(7.61)$ & $8.84(6.76)$ & $8.85(8.16)$ & $8.99(7.00)$ & 0.29 & 0.465 \\
\hline Practice nurse contacts & $6.07(8.07)$ & $5.26(7.76)$ & $6.28(8.98)$ & $5.92(9.83)$ & 0.45 & 0.245 \\
\hline Clinical readings* & $2.76(2.59)$ & $2.73(2.47)$ & $2.71(2.53)$ & $2.80(2.76)$ & 0.11 & 0.414 \\
\hline
\end{tabular}

*based on number of recordings of HbA1c, weight, blood oxygen, respiratory flow. 


\begin{tabular}{lccc}
$\begin{array}{l}\text { Table } 5 \text { Results of mixed models (data show incidence } \\
\text { rate ratio) }\end{array}$ & Model & Estimate $(95 \%$ Cl) & P \\
\hline $\begin{array}{l}\text { Endpoint } \\
\text { (interpretation) }\end{array}$ & Unadjusted & $1.05(0.90$ to 1.23$)$ & 0.520 \\
\hline $\begin{array}{l}\text { General practitioner } \\
\text { contacts }\end{array}$ & Adjusted & $1.04(0.95$ to 1.14$)$ & 0.404 \\
& $\begin{array}{c}\text { Combined } \\
\text { model }\end{array}$ & $1.04(0.90$ to 1.21$)$ & 0.560 \\
& Unadjusted & $1.14(0.81$ to 1.61$)$ & 0.438 \\
& Adjusted & $1.04(0.82$ to 1.30$)$ & 0.756 \\
Practice nurse contacts & Combined & $1.13(0.81$ to 1.58) & 0.468 \\
& model & & \\
& Unadjusted & $1.01(0.85$ to 1.20$)$ & 0.881 \\
& Adjusted & $1.00(0.90$ to 1.12$)$ & 0.931 \\
\hline
\end{tabular}

*based on number of recordings of $\mathrm{HbA1c}$, weight, blood oxygen, respiratory flow.

Our adjusted estimate suggested telehealth was associated with an increase of $4 \%$ in general practitioner consultations but this was not statistically significant on this sample. We also found no changes in the frequency with which clinical measurements such as weight, HbA1c, blood oxygen and respiratory flow were recorded on the general practice records. Given the frequency with which patients themselves recorded similar measurements using telehealth equipment (up to five times per week), our findings may indicate scope to improve the integration of information systems and care between the telehealth intervention and general practice.

\section{Strengths and limitations}

This particular study is part of a wider evaluation of the Whole Systems Demonstrator programme, which addresses a wider range of outcomes than in this paper. We hypothesised that telehealth could act in two opposing ways: either to increase or reduce the need for routine contact - though we found no overall effect it may be these two effects occurred but offset each other. Alternatively, it is possible that the impact of telehealth on workload was not manifested simply in the numbers of consultations but may be in the nature of consultations. For example, the content of general practice encounters might have changed following introduction of telehealth, even if the number of overall contacts did not change.

This study (with 2,317 patients included in the analysis) was much larger than the previous hypertension trial (which recruited 401 patients) [9]. However, it is possible that issues of sample size have contributed to the lack of statistical significance of the findings; we note we cannot rule out changes as great as a $10 \%$ reduction in general practitioner contacts or a $23 \%$ increase.

We relied on pre-existing, routine data sets. This has benefits over the alternative approach using selfreported data from patients, as patients do not always recall the use of health services accurately, particularly when it comes to events with low 'salience' including many general practice visits [19]. However, using existing administrative or clinical data sets may also bring problems with the completeness of data [20,21]. For example, problems with the completeness of coding meant that we were not able to separate surgery consultations from home visits. Similarly it may be that some tests may have stayed as written comments in the notes, and not been coded on the computer systems.

In a cluster-randomised trial there is a risk of selection bias. Although patients were blinded to treatment allocations until after they had consented to participate, it was not possible in this trial to guarantee that those people recruiting patients were always blinded. Therefore, there is a risk that patients with certain characteristics might be preferentially recruited into either the control or the intervention arm. We were reassured that we could observe few large differences between the characteristics of intervention and control patients at baseline, though as previously noted the number of participants per practice differed for intervention and control practices (medians 8.5 and 12). We were able to adjust for observed baseline characteristics using several forms of case-mix adjustment, and also applied a difference-in-difference estimator.

The study aimed to address a broad class of telehealth devices and did not aim to address specific devices and monitoring systems. Therefore these findings reflect a routine introduction of telehealth, which differed between sites based on a series of decisions by local teams. Thus, the monitoring systems used by the sites involved general practice staff to varying extents. Although this plurality may be seen as problematic to those wanting to replicate specific aspects of the interventions, in some ways it is the merit of a pragmatic trial as it meant we could reflect implementation decisions made by local teams.

The large, multicentre nature of this trial should improve the generalisability of the trial results. However, the patients and health care professionals who agree to participate in randomised controlled trials may differ from those who would use telehealth in routine clinical practice [22]. In this study, the participants were higher users of general practice services than other adult patients, but this was expected given that eligible patients had a long-term condition. A qualitative study that interviewed eligible patients who refused to participate in this trial found three broad reasons, including issues relating to the technology, self-care plans and perceived threats to existing services [23]. Also of interest is whether either of the treatments received in this trial (telehealth or control) might have differed from those offered in routine clinical practice. We note that staff in 
the three trial sites reported that the randomised nature of the trial constrained them, so that they were not able to innovate and improve the design of the telehealth trial in response to learning [24]. Further, telehealth devices are changing, and may have different impacts according to the nature of the surrounding services and context. Thus, the impact of telehealth in other settings might differ from that reported in this study.

The initial analysis of hospitalisation data found that emergency hospital admission rates appeared to increase for the control group following enrolment [12]. This leads us to suggest that either the trial protocol changed the management of patients allocated to this group, or that control patients may have reacted to their allocations, leading them to seek more care at the accident and emergency department. We note that, in the current study, we did not see a corresponding increase in general practice visits.

\section{Comparison with existing literature}

Most studies of telehealth that address impacts on service use focus on hospital activity [25]. Up until recently, studies that looked at primary care tended to focus on specific populations or issues, such as mental health problems or geographically-dispersed communities. The few studies that have considered similar forms of telehealth to those tested in this trial, have often reported more positive findings than here. For example, a feasibility study in 20 elderly patients indicated a time saving for the GP [6], while a study of home telemonitoring in heart failure patients found reductions in use of clinic visits [26]. However these studies were much smaller than this trial and for this reason may have recruited unrepresentative patients. Wade and colleagues commented on how telehealth alerts prompted frequent contacts and increased the case managers' workload [27]. A recent randomised study of telehealth in hypertension found approximately one additional GP surgery consultation and half a practice nurse surgery consultation per person in the intervention group compared with the usual care group [9], though in that study general practice teams were more directly involved with the telemonitoring than in the current study.

\section{Implications}

This study used linked operational data for large numbers of patients, a method that has considerable potential for future studies especially those that use retrospective analyses. The lack of observed impacts of telehealth suggests that fears about increases in general practice workload may be unfounded, at least when it comes to workload for clinical staff as measured by the number of contacts. The absence of any statisticallysignificant effects will not by itself provide a financial or workload incentive for primary care to deliver telehealth. However, under recent changes to the NHS, clinical commissioning groups including primary care professionals may have a firmer financial incentive to prevent hospital admissions.

Telehealth may have different impacts on general practice workload if designed differently. For example, we note that many of the measures captured by telehealth are recorded by general practices (such as weight and blood oxygen levels). In theory, this means there is potential for some work to be shifted from clinical staff to patients. However, we did not find that these readings were recorded in general practices any less frequently among the intervention group. Better information systems and higher general practice engagement might lead to more impressive changes. We note however, that clinical guidelines require some of these readings to be recorded in general practice and remuneration systems track the number of such measurements that are made. For diabetes, HbA1c is recognised as a more stable measure than blood glucose. This illustrates how the consequences of new technology depend on the wider health systems, and not only on the attributes of the technology itself.

\section{Conclusions}

In this study, the use of home based telehealth for people with chronic disease did not appear associated with changes in the frequency with which people contacted general practitioners and practice nurses. This suggest that fears that the widespread increase in the use of this technology may increases the burden on primary are unfounded. Conversely, we did not find evidence that telehealth led to a significant reduction in GP workload. We note that the way that telehealth impacts on primary care roles may be influenced by a number of other features in the health system. The challenge is to ensure that these systems lead to better integration of care than fragmentation.

\section{Additional file}

Additional file 1: Read codes used to define clinical readings.

\section{Competing interests}

All authors have completed the Unified Competing Interest form at http:// www.icmje.org/coi_instructions.html (available on request from the

corresponding author) and declare: no support from any organisation for the submitted work besides that described from the Department of Health; no financial relationships with any organisations that might have an interest in the submitted work in the previous 3 years; no other relationships or activities that could appear to have influenced the submitted work. A number of the authors undertake evaluative work funded by Government or public agencies but these do not create competing interests.

\section{Authors' contributions}

MB designed this element of the work, contributed to the analysis and wrote the first draft of the paper. AS contributed towards the design of the 
work, led on the collection of administrative data sets and conducted the analysis. HD contributed statistical advice. All authors revised the manuscript. All authors read and approved the final manuscript.

\section{Acknowledgements}

We thank all the study participants; staff from the three sites; and Bupa Health Dialog, for managing the remote collection of primary care data and providing predictive modelling analytic data files. The data analysis for this paper was generated using SAS software, version 9.3, copyright ( $2002-2010$ by SAS Institute Inc. SAS and all other SAS Institute Inc. product or service names are registered trademarks or trademarks of SAS Institute Inc., Cary, NC, USA. We would like to thank the wider Whole System Demonstrator Evaluation Team: James Barlow, Michelle Beynon, John Billings, Peter Bower, Martin Cartwright, Jennifer Dixon, Ray Fitzpatrick, Catherine Henderson, Jane Hendy, Shashi Hirani, Martin Knapp, Virginia McNeill, Lorna Rixon, Anne Rogers, Caroline Sanders and Stanton Newman (Principal Investigator). The Evaluation Team have contributed to discussions about the design and practicalities of the study throughout the project; Professor Stanton Newman provided helpful comments on an earlier version of this paper.

\section{Funding}

The study was funded by the Department of Health. The Department of Health reviewed the protocol for the study and provided project manage support for the implementation of telehealth. Data collection and analysis was undertaken by the named authors who made the final editorial decisions about the manuscript. The views expressed are those of the authors and are not necessarily those of the Department of Health.

\section{Author details}

${ }^{1}$ The Nuffield Trust, 59 New Cavendish Street, W1G 7LP London, UK ${ }^{2}$ Norwich Medical School, University of East Anglia, Chancellors Drive, NR4 7TJ Norwich, UK. ${ }^{3}$ Oxford Outcomes, Seacourt Tower, West Way, OX2 0J Oxford, UK.

Received: 29 July 2013 Accepted: 30 September 2013

Published: 8 October 2013

\section{References}

1. Department of Health: Whole system demonstrator programme: headline findings. 2011. Available at: www.dh.gov.uk/prod_consum_dh/groups/ dh_digitalassets/documents/digitalasset/dh_131689.pdf [accessed 201207 23]

2. Henderson C, Knapp M, Fernandez J-L, Beecham J, Hirani SP, Cartwright M, Rixon $L$, et al: Cost effectiveness of telehealth for patients with long term conditions (whole systems demonstrator telehealth questionnaire study): nested economic evaluation in a pragmatic, cluster randomised controlled trial. BMJ 2013, 346(mar20 4):f1035. doi:10.1136/bmj.f1035.

3. May CR, Finch TL, Cornford J, Exley C, Gately C, Kirk S, Jenkings KN, Osbourne J, Robinson AL, Rogers A, Wilson R, Mair FS: Integrating telecare for chronic disease management in the community: what needs to be done? BMC Health Serv Res 2011, 11:131.

4. Mclean S, Protti $D$, Sheik A: Telehealthcare for long term conditions. BMJ 2011, 342:d120. doi:10.1136/bmj.d120.

5. Terschüren C, Fendrich $K$, van den Berg N, Hoffmann W: Implementing telemonitoring in the daily routine of a GP practice in a rural setting in northern Germany. J Telemed Telecare 2007, 13(4):197-201.

6. Roberts A, Garrett Lm Godeen DJ: Can telehealth deliver for rura Scotland? Lessons from the Argyll \& Bute telehealth programme. Scott Med J 2012, 57:33-37. doi:10.1258/smj.2011.011288.

7. Martín-Lesende I, Orruño E, Bilbao A, Vergara I, Cairo1 C, Bayón JC, Reviriego E, Romo MI, Larrañaga J, Asua J, Abad R, Recalde E: Impact of telemonitoring home care patients with heart failure or chronic lung disease from primary care on healthcare resource use (the TELBIL study randomised controlled trial). BMC Health Serv Res 2013, 13:118.

8. Lewis KE, Annandale JA, Warm DL, Rees SE, Hurlin C, Blyth H, Syed Y, Lewis $L$ : Does home telemoniroting after pulmonary rehabilitation reduce healthcare use in optimized COPD? A pilot randomised trial. J Chronic Obstructive Pulm Dis 2010, 7:44-50.

9. McKinstry B, Hanley J, Wild S, Pagliari C, Paterson M, Lewis S, Sheikh A, Krishan A, Stoddart A, Padfield P: Telemonitoring based service redesign for the management of uncontrolled hypertension: multicentre randomised controlled trial. BMJ 2013, 346:f3030. doi:10.1136/bmj.f3030 (Published 24 May 2013)

10. Stoddart A, Hanley J, Wild S, Pagliari C, Paterson M, Lewis S, Sheikh A, Krishan A, Padfield P, McKinstry B: Telemonitoring-based service redesign for the management of uncontrolled hypertension (HITS): cost and costeffectiveness analysis of a randomised controlled trial. BMJ Open 2013, 3(5). doi:10.1136/bmjopen-2013-002681.

11. Bower P, Cartwright M, Hirani SP, Barlow J, Hendy J, Knapp M, et al: A comprehensive evaluation of the impact of telemonitoring in patients with long-term conditions and social care needs: protocol for the whole system demonstrator cluster randomised trial. BMC Health Serv Res 2011, 11:184

12. Steventon A, Bardsley M, Billings J, Dixon J, Doll H, Hirani S, Cartwright M, Rixon L, Knapp M, Henderson C, Rogers A, Fitzpatrick R, Hendy J, Newman $S$ : Effect of telehealth on use of secondary care and mortality: findings from the whole system demonstrator cluster randomised trial. BMJ 2012, 344:e3874

13. Cartwright M, Hirani SP, Rixon L, Beynon M, Doll H, Bower P, Bardsley M, et al: Effect of telehealth on quality of life and psychological outcomes over 12 months (whole systems demonstrator telehealth questionnaire study): nested study of patient reported outcomes in a pragmatic, cluster randomised controlled trial. BMJ 2013, 346(feb26 2):f653-f653. doi:10.1136/bmj.f653.

14. Flury BK, Reidwyl $\mathrm{H}$ : Standard distance in univariate and multivariate analysis. Am Stat 1986, 40:249-251.

15. Normand ST, Landrum MB, Guadagnoli E, Ayanian JZ, Ryan TJ, Cleary PD, et al: Validating recommendations for coronary angiography following acute myocardial infarction in the elderly: a matched analysis using propensity scores. J Clin Epidemiol 2001, 54(4):387-398.

16. Wennberg D, Siegel M, Darin B, et al: Combined predictive model: final report and technical documentation. London: Kings Fund; 2006. Available at: http:// www.kingsfund.org.uk/sites/files/kf/field/field_document/PARR-combinedpredictive-model-final-report-dec06.pdf.

17. Campbell MK, Grimshaw JM, Elbourne GR: Intracluster correlation coefficients in cluster randomized trials: empirical insights into how they should be reported. BMC Med Res Methodo/ 2004, 4. doi:10.1 186/1471-2288-4-9.

18. Hippisley-Cox J, Vinogradova Y: Trends in consultation rates in general practice 1995 to 2008:analysis of the QResearch ${ }^{\circledR}$ database. Final report to the NHS Information Centre and Department of Health. Leeds: The Health and Social Care Information Centre; 2009.

19. Bellón J a, Lardelli P, Luna JD, Delgado a: Validity of self reported utilisation of primary health care services in an urban population in Spain. J Epidemiol Community Health 2000, 54(7):544-551.

20. Lezzoni LI: Assessing quality using administrative data. Ann Intern Med 1997, 127:666-674.

21. Roos LL, Mustard CA, Nicol JP, McLerran DF, Malenka DJ, Young TK, Cohen MM: Registries and administrative data: organization and accuracy. Med Care 1993, 31:201-212.

22. Rothwell PM: External validity of randomised controlled trials: "to whom do the results of this trial apply?". Lancet 2005, 365:82-93.

23. Sanders C, Rogers A, Bowen R, Bower P, Hirani S, Cartwright M, et al: Exploring barriers to participation and adoption of telehealth and telecare within the whole system demonstrator trial: a qualitative study. BMC Health Serv Res 2012, 12:220

24. Hendy J, Chrysanthaki T, Barlow J, Knapp M, Rogers A, Sanders C, et al: An organisational analysis of the implementation of telecare and telehealth: the whole systems demonstrator. BMC Health Serv Res 2012, 12(1):403.

25. Clark RA, Inglis SC, MCAlister FA, Cleland JGF, Stewart S: Telemonitoring or structured telephone support programmes for patients with chronic heart failure: systematic review and meta-analysis. BMJ 2007, 334:942

26. Dar O, Riley J, Chapman C, Dubrey SW, Morris S, Rosen SD, Roughton M, Cowie MR: A randomized trial of home telemonitoring in a typical elderly heart failure population in north west London: results of the home-HF study. Eur J Heart Fail 2009, 11(3):319-325. Epub 2009 Jan 27.

27. Wade MJ, Desai AS, Spettell CM, Snyder AD, McGowan-Stackewicz V, Kummer PJ, Maccoy MC, Krakauer RS: Telemonitoring with case management for seniors with heart failure. Am J Manag Care 2011, 17(3):e71-79.

doi:10.1186/1472-6963-13-395

Cite this article as: Bardsley et al:: Impact of telehealth on general practice contacts: findings from the whole systems demonstrator cluster randomised trial. BMC Health Services Research 2013 13:395. 